\title{
Label-free exonuclease I-assisted signal amplification colorimetric sensor for highly sensitive detection of kanamycin
}

\author{
Jingwen Li ${ }^{a}$, Yongming Liu ${ }^{a}$, Hao Lin ${ }^{a}$, Yan Chen ${ }^{\mathrm{b}}$, Zhenbo Liu ${ }^{\mathrm{a}}$, Xuming Zhuang ${ }^{\mathrm{a}}$, \\ Chunyuan Tian ${ }^{\mathrm{a}}$, Xiuli Fu ${ }^{\text {a, }}$, Lingxin Chen ${ }^{\mathrm{c}, \mathrm{d}, *}$ \\ ${ }^{a}$ School of Chemistry and Chemical Engineering, Yantai University, Yantai 264005, China \\ ${ }^{\mathrm{b}}$ College of Resources and Environmental Engineering, Shandong Agriculture and Engineering University, Jinan 250100, China \\ ${ }^{\mathrm{c}}$ CAS Key Laboratory of Coastal Environmental Processes and Ecological Remediation, The Research Center for Coastal Environmental Engineering and Technology, \\ Yantai Institute of Coastal Zone Research, Chinese Academy of Sciences, Yantai 264003, China \\ d College of Chemistry and Chemical Engineering, Qufu Normal University, Qufu 273165, China
}

\section{A R T I C L E I N F O}

\section{Keywords:}

Kanamycin

Exonuclease I

Colorimetric

Protamine

Gold nanoparticles

\begin{abstract}
A B S T R A C T
A label-free colorimetric method based on exonuclease I (Exo I)-assisted signal amplification with protamine as a medium was developed for analysis of kanamycin. In this study, a double-stranded DNA (dsDNA) probe was tailored by manipulating an aptamer and its complementary DNA (cDNA) ensuring detection of target with high selectivity and excellent sensitivity. Herein, protamine could not only combine with negatively charged gold nanoparticles but also interaction with polyanion DNA. Upon addition of target kanamycin, the target-aptamer complex was formed and the cDNA was released. Thus, both aptamer and cDNA could be digested by Exo I, and the captured kanamycin was liberated for triggering target recycling and signal amplification. Under optimized conditions, the proposed colorimetric method realized a low detection limit of $2.8 \times 10^{-14} \mathrm{M}$ along with a wide linear range plus excellent selectivity. Our strategy exhibited enormous potentials for fabricate various kinds of biosensors based on target-induced aptamer configuration changes.
\end{abstract}

\section{Introduction}

Aminoglycoside antibiotics have been widely utilized in the field of prevention and treatment of various diseases as well as promotion the animal growth (Qin et al., 2017; Wang, Wang, Wang, \& Chen, 2017; Zhu, Chandra, Song, Ban, \& Shim, 2012). As a common aminoglycoside antibiotic, kanamycin has been extensively used in the veterinary medicine to treat gram-positive and gram-negative infections due to its strong spectrum activity and low cost (Han et al., 2017; Li et al., 2017; Ma, Sun, Tu, Zhang, \& Diao, 2017; Xu et al., 2015; Saratale et al., 2020). However, the incorrect/uncontrolled use of kanamycin may lead to its residues in animal derived food, which resulting in ultimate accumulation in human body (Long, Zhang, Yang, Zeng, \& Jiang, 2015; Zhou, Zhang, \& Tian, 2014) further causing serious side effects such as ototoxicity and nephrotoxicity. Owing to the great threats to human health (Abnous et al., 2017; Luo et al., 2015), maximum-residue levels (MRLS) for kanamycin has been established by European Union (150 $\mu \mathrm{g}$ $\mathrm{kg}^{-1}$ in milk, $100 \mu \mathrm{g} \mathrm{kg}^{-1}$ in meat, $600 \mu \mathrm{g} \mathrm{kg}^{-1}$ in liver and $2500 \mu \mathrm{g} \mathrm{kg}^{-1}$ in kidney) (Ma et al., 2017). To date, various approaches have been reported for determination of kanamycin in food products or biological samples, such as high performance liquid chromatography (Abu-Qare \& Abou-Donia, 2001; Blanchaert, Poderós Jorge, Jankovics, Adams, \& Van Schepdael, 2013), capillary electrophoresis (García-Ruiz et al., 2005; Hernández, Borrull, \& Calull, 2003) gas chromatography (Berijani, Assadi, Anbia, Milani Hosseini, \& Aghaee, 2006) and enzyme-linked immunosorbent assay (Chen et al., 2008; Yu et al., 2013). Although these methods have made great progress in detection of kanamycin, the requirement of time-consuming sample preparation, tedious operation procedures, high consumption of various reagents or sophisticated equipment has seriously limit their further applications. Therefore, it is urgent to develop simple, convenient and sensitive strategy for the determination of kanamycin in foodstuff.

Colorimetric technique has been paid close attention in chemical and biological sensing, because it could be easily visual judgment by naked

\footnotetext{
* Corresponding authors at: School of Chemistry and Chemical Engineering, Yantai University, Yantai 264005, China (X. Fu). CAS Key Laboratory of Coastal

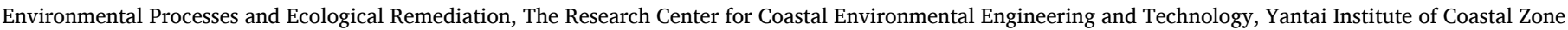
Research, Chinese Academy of Sciences, Yantai 264003, China (L. Chen).

E-mail addresses: fx11984.happy@163.com (X. Fu), 1xchen@yic.ac.cn (L. Chen).
} 
eye observation, has simple operation process, and avoids the need of sophisticated equipment (Liu \& Lu, 2006; Sabela, Balme, Bechelany, Janot, \& Bisetty, 2017). Especially, gold nanoparticles (AuNPs) based colorimetric methods have earned tremendous momentum due to their excellent localized surface plasmon resonance and high extinction coefficient (Cao \& Li, 2011; Shahdordizadeh et al., 2018). Based on the changes in the absorbance of AuNPs from dispersion to aggregation, and color changes from wine red to purple or blue, researchers have promoted AuNPs-based colorimetry for detection of various species, including metal ions (Liu et al., 2015), DNA (Yang, Jockusch, Vicens, Turro, \& Tan, 2005) small molecules (Wang, Chen, Wang, \& Tan, 2017) and proteins (Chen et al., 2014).

Compared to antibodies, aptamers exhibit outstanding merits, such as excellent stability, in vitro synthesis, easy of modification and fix, small size (Chen, Xiong, Yu, \& Lai, 2019), low toxicity and low cost. Given their unique advantages, aptamers have been broadly used as recognition element to construct aptamer-based biosensors. In this case, numerous aptasensors have been developed in the fields of food safety, environmental monitoring and clinical diagnosis, based on colorimetric (Han et al., 2017), fluorescence (Xing et al., 2016), electrochemical (Zhang et al., 2019), chemiluminescence (Hao, Gu, Duan, Wu, \& Wang, 2016) and surface plasmon resonance (Tu, Sun, \& Grattan, 2014).

In order to obtain high sensitivity, different signal amplification techniques are used in aptamer-based colorimetric biosensors (Ramezani, Danesh, Lavaee, Abnous, \& Taghdisi, 2016), such as hybrid chain reactions (HCR) (Blanchaert et al., 2013), polymerase chain reactions (PCR) (Xu, Ying, \& Ping, 2019), tool enzymes (Ma et al., 2018) and roll ring amplification (RCA) (Liu et al., 2013). Among these signal amplification strategies, the signal amplification technology based on tool enzyme assisted has been rapidly developed in biochemical analysis in recent years due to its merits of simple operation, high sensitivity and specificity, as well as relatively short reaction time (Liu et al., 2013). Among these signal amplification strategies, nuclease-assisted signal amplification technology designed by combining the cycle effect of nuclease digestion, aptamer and nanotechnology has been applicated frequently in biochemical analysis due to its merits of simple operation, high sensitivity and specificity, as well as relatively short reaction time (Liu et al., 2013). There are two main types of signal amplification strategies based on nuclease assisted signal amplification (Chen et al., 2018; Zhang et al., 2019): one is the sequence dependent signal amplification strategy with restriction enzyme or restriction enzyme assisted signal amplification; the other is the signal amplification strategy without sequence dependent nuclease assisted signal amplification. In terms of the universality of nuclease-assisted signal amplification strategy, the exonuclease without sequence dependence is simpler and more feasible in the aspect of probe design and target analysis, thus which shows a broader application prospect.

Herein, in this study, a simple, sensitive and specific kanamycin colorimetric sensor was developed based on exonuclease I (Exo I)assisted signal amplification and protamine mediated aggregation of AuNPs. The introduction of aptamer as recognition element ensures the specificity of this strategy owing to its strong affinity for the target molecule. In general, the target binds to the aptamer at a ratio of 1:1, which means one target can be recognized and bound by one aptamer. Fortunately, Exo I break this boundary which can specifically shear the single stranded cDNA as well as the kanamycin aptamer that bind to the target, destroying the structure of the target-aptamer complex, thus releasing the target to participate in the new binding cycle. In this case, the signal could be significantly amplified, and thus make the detection more sensitive and efficient. Positively charged protamine is a polycation rich in basic arginine residues, which can not only electrostatically combine with negatively charged DNA, but also interact with negatively charged AuNPs. And the interaction between protamine and AuNPs can induce the aggregation of AuNPs, thereby causing changes in the surface plasmon resonance (SPR) absorptions. Our Exo I-assisted signal amplification colorimetric assay only needs simple mixing of the
dsDNA probe, target, protamine and AuNPs in homogeneous solution, without containing modification, or separation steps. By altering the aptamer sequence, it can be easily extended to detect other targets, which demonstrating that this method has a great potential as a valuable tool for screening multiple antibiotic residues in food safety.

\section{Experimental section}

\subsection{Reagents and chemicals}

Protamine sulfate salt, tetrachloroauric (III) acid tetrahydrate $\left(\mathrm{HAuCl}_{4} \cdot 4 \mathrm{H}_{2} \mathrm{O}\right)$, and sodium citrate $\left(\mathrm{C}_{6} \mathrm{H}_{5} \mathrm{Na}_{3} \mathrm{O}_{7} \cdot 2 \mathrm{H}_{2} \mathrm{O}\right)$ were purchased from Sigma-Aldrich (USA). Kanamycin sulfate, tetracycline hydrochloride, p-hydroxy-ampicillin, chloramphenicol, ampicillin and gentamicin sulphate were obtained from Sangon Biotechnology Inc. (Shanghai, China). Kanamycin aptamer ( $5^{\prime}$-TGG GGG TTG AGG CTA AGC CGA-3') and CDNA ( $5^{\prime}$-TCG GCT TAG CCT CAA- $3^{\prime}$ ) were synthesized by Sangon Biotechnology Inc. (Shanghai, China). Sodium chloride ( $\mathrm{NaCl}$ ), sodium borohydride $\left(\mathrm{NaBH}_{4}\right)$, magnesium chloride $\left(\mathrm{MgCl}_{2}\right)$, glucose, and absolute ethyl alcohol were obtained from Sinopharm Chemical Reagent Co., Ltd. (Shanghai, China). Exonuclease I was purchased from New England Biolabs (Beijing, China). All other chemicals were of analytical reagent grade or higher and used without further purification. Double distilled water (18.2 $\mathrm{M} \Omega$, Pall Cascada) was used throughout the experiments.

\subsection{Instrumentation}

SPR absorption spectra were recorded by using a Thermo Scientific NanoDrop 2000/2000C spectrophotometer (USA). The transmission electron microscopy (TEM) images were acquired using a JEM-1400 electron microscope (Japan) with an accelerating voltage of $100 \mathrm{kV}$. Dynamic light scattering (DLS) measurement was carried out with a Nano-ZS90 instrument (Malvern, UK).

\subsection{Preparation of nanoparticles}

AuNPs were prepared by the sodium citrate reduction of $\mathrm{HAuCl}_{4}$ method with necessary modification (Liu \& Lu, 2006). Prior to the synthesis experiment, all the glassware used was thoroughly soaked in aqua regia ( 3 parts $\mathrm{HCl}, 1$ parts $\mathrm{HNO}_{3}$ ), rinsed with ultrapure water, and oven-dried before using. Typically, $100 \mathrm{~mL}$ aqueous solution consisting of $1 \mathrm{mM} \mathrm{HAuCl}_{4}$ was heated with vigorous stirring in a three-necked round-bottomed flask equipped with a reflux condenser. After boiling, $10 \mathrm{~mL}$ of $38.8 \mathrm{mM}$ sodium citrate solution was added rapidly to the above solution. The color of the solution changed from yellow to light gray, and finally to wine red within $15 \mathrm{~min}$. Finally, the resultant AuNPs was cooled to room temperature and stored at $4{ }^{\circ} \mathrm{C}$ for further use. The absorption spectra, TEM image and DLS data were used to determine the average size of AuNPs (Fig. 2, Fig. S1). The size of the prepared AuNP is estimated to be $13 \pm 2 \mathrm{~nm}$. AuNPs with different sizes $(28 \mathrm{~nm}, 31 \mathrm{~nm}$ and $42 \mathrm{~nm}$ ) could be obtained by adjusting the volumes of $38.8 \mathrm{mM}$ trisodium citrate, including 8,6 and $4 \mathrm{~mL}$, respectively (Figs. $\mathrm{S} 2$ and S3). And $8 \mathrm{~nm}$ AuNPs was prepared by the reduction of $\mathrm{HAuCl}_{4}$ with $\mathrm{NaBH}_{4}$ at room temperature following a previous method with slight modification. (Obare, Hollowell, \& Murphy, 2002)

\subsection{Colorimetric determination of kanamycin}

In this experiment, the kanamycin-aptamer binding buffer was 10 mM HEPES buffer solution ( $\mathrm{pH} 7.4$ ) containing $50 \mathrm{mM} \mathrm{NaCl}$ and $2.5 \mathrm{mM}$ $\mathrm{MgCl}_{2}$. The various concentrations of standard kanamycin solution and aptamer solution were prepared with binding buffer.

For kanamycin analysis, the aptamer/cDNA double-stranded structure (dsDNA) probe was prepared according to the following procedure: $10 \mu \mathrm{L}$ of $10 \mu \mathrm{M}$ kanamycin aptamer and $10 \mu \mathrm{L}$ of $10 \mu \mathrm{M}$ cDNA were 
initially mixed with binding buffer. Then, the resulting mixture was heated to $95{ }^{\circ} \mathrm{C}$ for $5 \mathrm{~min}$ and finally slowly cooled to room temperature within $2 \mathrm{~h}$. Next, $5 \mu \mathrm{L}$ of dsDNA probe $(1.5 \mu \mathrm{M})$ and $5 \mu \mathrm{L}$ of Exo I (1500 U $\mathrm{mL}^{-1}$ ) were added into $40 \mu \mathrm{L}$ kanamycin standards with different concentrations. The mixture was incubated for $1.5 \mathrm{~h}$ to execute Exo Iassisted target recycling. Subsequently, $100 \mu \mathrm{L}$ of protamine $(1.5 \mu \mathrm{g}$ $\mathrm{mL}^{-1}$ ) was added to the above solution and incubated for $10 \mathrm{~min}$. Finally, $400 \mu \mathrm{L}$ AuNPs was added to the above solution and incubated for another $2 \mathrm{~min}$. The SPR absorption spectra of the mixture were recorded for quantitative analysis.

\subsection{Detection of kanamycin in milk}

Milk samples were obtained from local supermarkets. Milk samples are processed according to the method described by Zhang and Zhou (Zhou et al., 2014). Firstly, the acetic acid solution (20\%, V/V) was drop wised to the milk sample until the $\mathrm{pH}$ was adjusted to 4.6 , so that the protein in the milk (mainly tyrosine) was denatured and precipitated at the isoelectric point. Then, the sample was placed in a water bath at $45^{\circ} \mathrm{C}$ for $10 \mathrm{~min}$ to ensure that the protein was completely precipitated. After that, the sample was centrifuged at $9580 \times g$ for $30 \mathrm{~min}$, followed by the removal of the precipitate containing denatured protein and fat. Subsequently, the supernatant was filtered through a $0.22 \mu \mathrm{m}$ ultrafiltration membrane to remove lipids and the filtrates were regulated to neutral $\mathrm{pH}$ for further detection. Add a certain amount of kanamycin to the pre-treated milk sample, and then perform the kanamycin colorimetric detection according to the above steps.

\section{Results and discussion}

3.1. Design principle of detection kanamycin based on aptamer structure switch colorimetric sensor using Exo I as a signal amplifier

The construction of target-binding induced aptamer structure switch colorimetric sensor was auxiliary with the strategy of Exo I-assisted signal amplification and polycationic protamine mediated aggregation of negatively charged citric-coated AuNPs. The sensing mechanism was shown in Fig. 1. In our design, the electrostatic interaction between protamine and DNA is strongly limited by the length of the DNA strand. Exo I-assisted signal amplification was initiated due to the introduction of target kanamycin. Exo I is a kind of enzyme that specifically hydrolyzes phosphodiester bond from $3^{\prime}$ to $5^{\prime}$ in sequence, and the final product is a single nucleotide. In the presence of kanamycin, kanamycin specifically bound to the kanamycin aptamer, followed by dissociation with cDNA to form single strand that provided catalytic substrate for Exo I. In this case, the single stranded kanamycin aptamer and cDNA were hydrolyzed into small fragments by Exo I, accompanying failing to interact with protamine owing to the relatively weak electrostatic interaction. And the released target could be recycled during the cycle of bonding-hydrolysis-release. Together with the occurrence of targetbinding induced aptamer structure switch and the Exo I-assisted signal amplification, the AuNPs could interaction with the remaining free protamine, which resulting in the aggregation of AuNPs with a color change from red to blue (Fig. 2). However, in the absence of kanamycin,
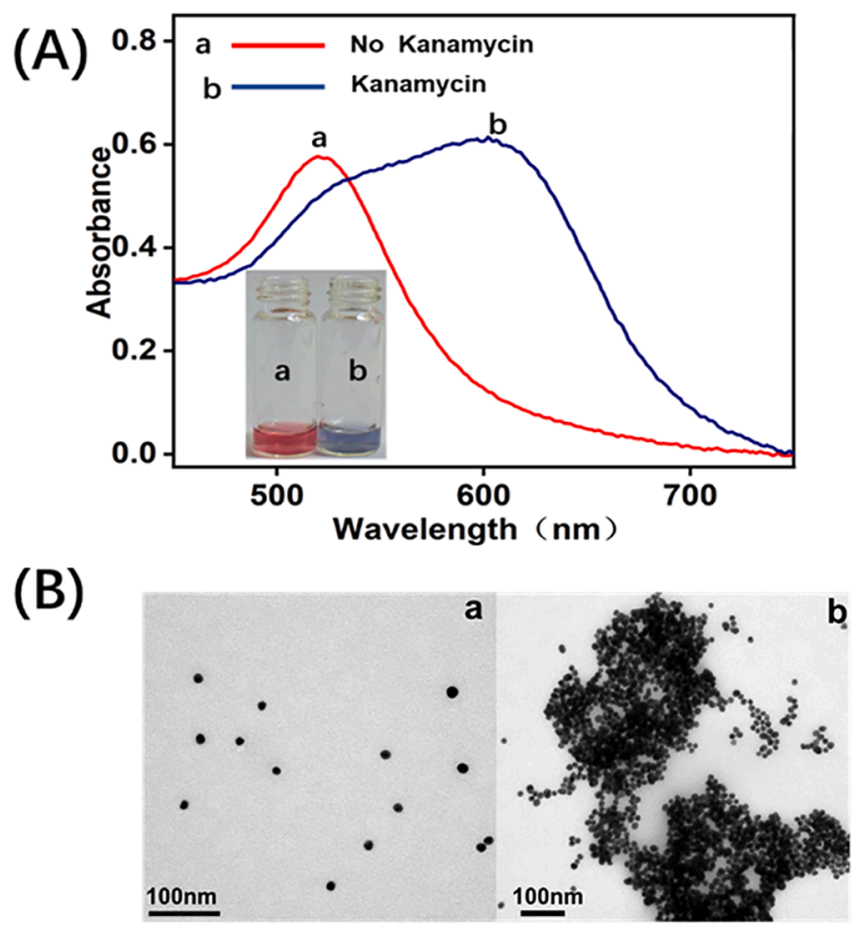

Fig. 2. (A) Absorption spectra and (B) corresponding TEM images of AuNPs in the absence (a) and presence (b) of kanamycin.
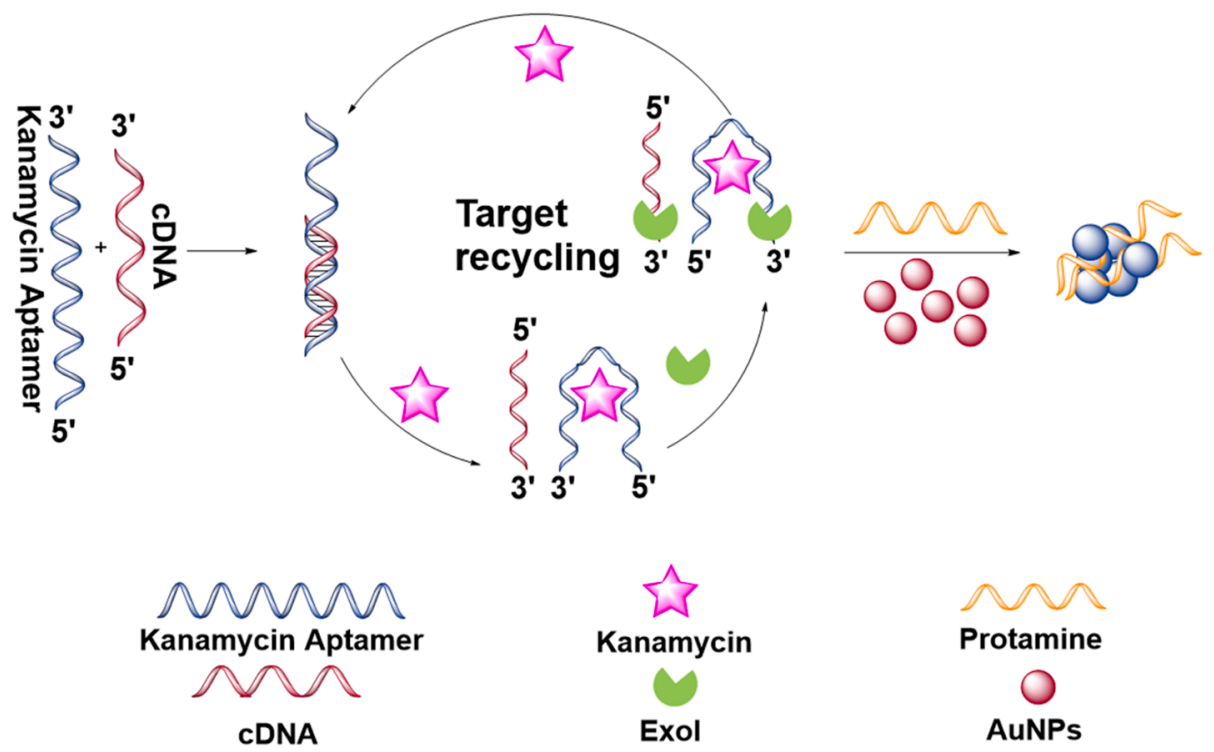

Fig. 1. Principle of the label-free Exo I-assisted signal amplification colorimetric sensor for highly sensitive detection of kanamycin. 
the aptamer-cDNA dsDNA probe could preferentially interact with protamine, thus the AuNPs existed in a dispersed state with native color. Finally, the quantitative analysis of kanamycin could be achieved by measuring the change of SPR absorption of AuNPs.

\subsection{Electrophoresis characterization}

Agarose gel electrophoresis was employed to verify the catalytic activity of Exo I toward anti-kanamycin aptamer upon binding kanamycin. As shown in Fig. S4, lanes 1, 2 and 4 presented a bright band, whereas the lane 3, owing to the hydrolysis of the anti-kanamycin aptamer and/or cDNA by Exo I and then migrated out of the gel. This result confirms that the kanamycin aptamer can be cleaved upon binding kanamycin, which is consistent with the previous studies (Chen et al., 2018; Zeng, Tang, Zhang, Luo, \& Tang, 2018).

\subsection{Optimization of experimental conditions}

The concentration of protamine severely restricted the degree of aggregation of AuNPs and the amount of dsDNA probes. Therefore, the effect of protamine concentration on the analytical performance was first optimized. As shown in Fig. S5A, the ratio of SPR absorption value at $650 \mathrm{~nm}$ to $520 \mathrm{~nm}\left(A_{650} / A_{520}\right)$ increased with the increase of the protamine concentration up to $1.5 \mu \mathrm{g} \mathrm{mL}^{-1}$, while higher concentration of protamine did not obvious increased the ratio of SPR absorption. This occurred because the aggregation degree of AuNPs increased with the increase of the protamine concentration lower than $1.5 \mu \mathrm{g} \mathrm{mL} L^{-1}$, and further increasing the concentration, the aggregation degree was close to the maximum. Consequently, $1.5 \mu \mathrm{g} \mathrm{mL}^{-1}$ was chosen as the optimum protamine concentration.

In addition, the concentration of dsDNA probe was explored for improving the sensitivity. As revealed in Fig. S5B, the higher the concentration of dsDNA probe in the range of 0 to $2 \mu \mathrm{M}$, the smaller the ratio of $A_{650} / A_{520}$ was obtained. This may be due to the fact that with the increase of dsDNA concentration, more and more dsDNA was combined with protamine, which leads to the gradually recovery of AuNPs to its original dispersed state. However, by further increasing the concentration of dsDNA, the ratio $A_{650} / A_{520}$ is almost the same as that in the presence of $1.5 \mu \mathrm{M}$ dsDNA. Therefore, the optimal concentration of dsDNA probe is $1.5 \mu \mathrm{M}$.

Next, the influence of the amount of Exo I on the signal amplification for Exo I-assisted target recycling was studied with five different concentrations. As illustrated in Fig. S6, it could be seen that the ratio of $A_{650} / A_{520}$ of AuNPs gradually increased with increasing the concentration of Exo I owing to the enhanced signal amplification. When the concentration reached $1500 \mathrm{U} \mathrm{mL}^{-1}$, our strategy displayed almost the highest ratio value. Thus, $1500 \mathrm{U} \mathrm{mL}^{-1}$ was chosen as the optimal concentration of Exo I.

Finally, the effect of the size of AuNPs was investigated. Five different diameters of AuNPs were tested including $8 \mathrm{~nm}, 13 \mathrm{~nm}, 28 \mathrm{~nm}$, $31 \mathrm{~nm}$ and $42 \mathrm{~nm}$. As shown in Fig. S7, the smaller the diameter of AuNPs in the range of $13-42 \mathrm{~nm}$, the higher the sensitivity of our colorimetric sensor was for analysis of kanamycin. However, further decreasing the diameter of AuNPs would induce lower sensitivity. Thus, the diameter of $13 \mathrm{~nm}$ was chosen.

\subsection{Sensitivity and selectivity for the detection of kanamycin}

To assess the sensitivity, our proposed Exo I-assisted signal amplification strategy was applied for quantitative analysis of kanamycin under optimal conditions. As shown in Fig. 3A, with the increase of kanamycin concentration, the SPR absorption band of AuNPs at $520 \mathrm{~nm}$ region decreased while it increased at $650 \mathrm{~nm}$. At the same time, the color change of AuNPs from red to blue was displayed, as can be seen in Fig. 3A. In this case, the two SPR absorption values at $520 \mathrm{~nm}$ and 650 $\mathrm{nm}$ were used to represent the relative amounts of dispersed and
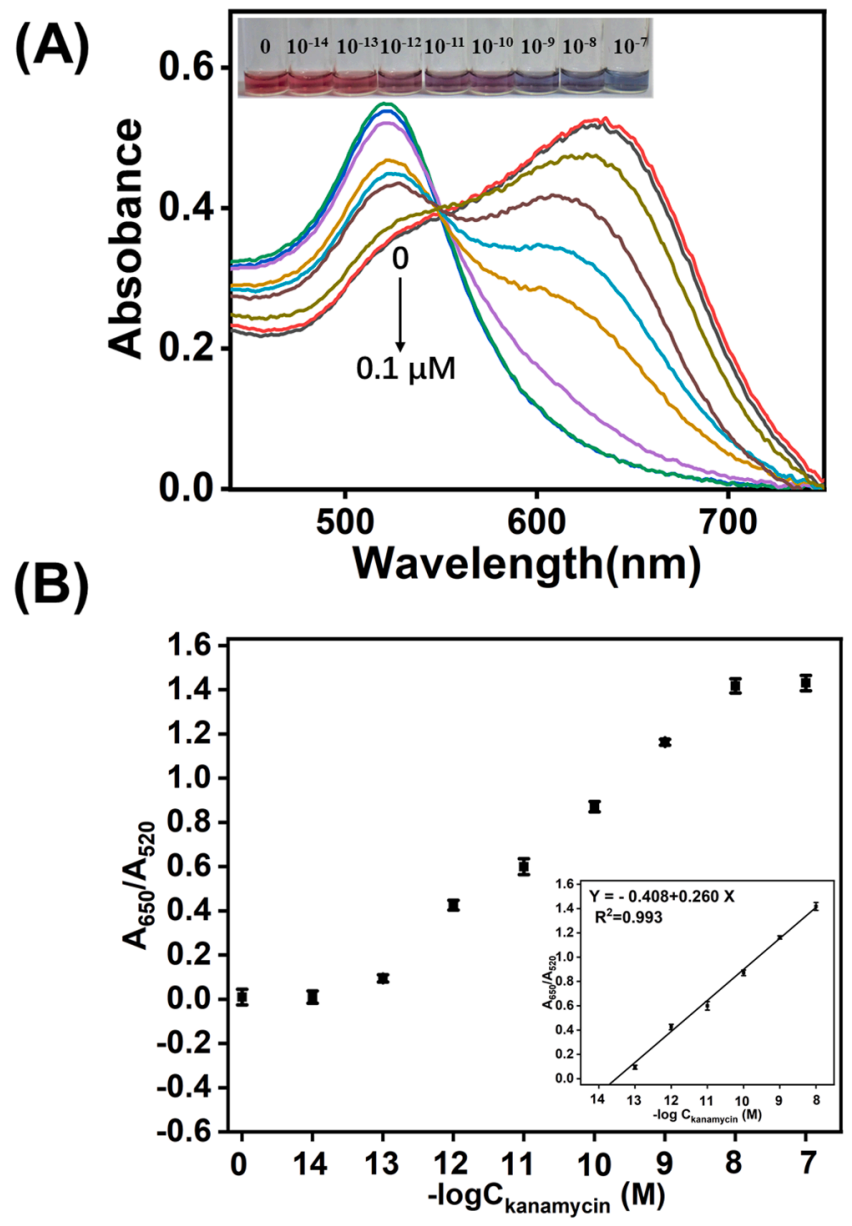

Fig. 3. (A) Absorption spectra (inset photographic images were the corresponding colorimetric response) of AuNPs in the system upon incubation with different concentrations of kanamycin (from left to right: $0,10^{-14}, 10^{-13}$, $10^{-12}, 10^{-11}, 10^{-10}, 10^{-9}, 10^{-8}, 10^{-7} \mathrm{M}$, respectively). (B) The plot of $A_{650}$ ' $A_{520}$ values versus the concentrations of kanamycin in 10 mM HEPES ( $\mathrm{pH} 7.4$ ) buffer solution. Inset: the corresponding calibration curve of the Exo I-assisted system for detection of kanamycin. Error bars indicate standard deviations of five measurements.

aggregated AuNPs. Fig. 3B showed that the absorption ratio is linear in the range of $10^{-13}$ to $10^{-8} \mathrm{M}$ in the concentration range of kanamycin. The limit of detection (LOD) value was calculated to be $2.8 \times 10^{-14} \mathrm{M}$ according to the $3 \sigma / s$ method (LOD $=3 \sigma / s, \sigma$ is the standard deviation of the blank sample, and $s$ is the slope of the standard curve), which is at least 1 orders of magnitude lower than that of reported colorimetry methods as well as other detection methods (Table S1) (Blanchaert et al., 2013; Chen et al., 2018; Han et al., 2019; Hao et al., 2016; Ramezani et al., 2016; Saratale et al., 2020; Xu et al., 2015; Yu et al., 2013).

To evaluate the selectivity of the developed strategy for kanamycin detection, the possible interfering agents including glucose, p-hydroxyampicillin, chloramphenicol, tetracycline hydrochloride, ampicillin and gentamicin sulphate were selected to test. As shown in Fig. 4, glucose, phydroxy-ampicillin, chloramphenicol, tetracycline hydrochloride, ampicillin and gentamicin sulphate did not cause obvious absorption ratio value increase. Only in the presence of kanamycin, the absorption ratio value was significantly enhanced, which indicated the strong interaction between kanamycin and the aptamer. These results demonstrated that our developed strategy provided attractive specificity toward kanamycin. 


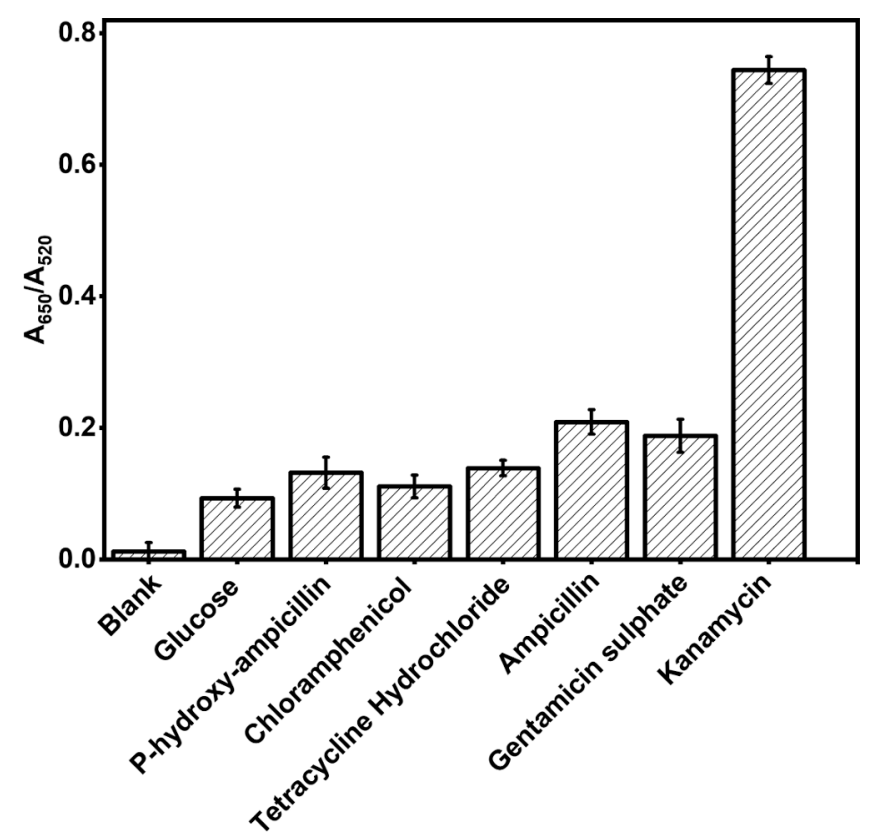

Fig. 4. Selectivity assay of the Exo I-assisted system upon the addition of kanamycin $\left(10^{-10} \mathrm{M}\right)$ and various potentially coexisting species $\left(10^{-7} \mathrm{M}\right)$. The error bars represent standard deviations from five parallel measurements.

\subsection{Application to real milk samples}

In order to further evaluate the potential application of the proposed atpasensor in real samples, milk samples were spiked with various concentrations of kanamycin. As shown in Fig. S8, the LOD for kanamycin was $5.5 \times 10^{-14} \mathrm{M}$ in milk samples with a wide dynamic linear range from $10^{-13}$ to $10^{-8} \mathrm{M}$, which is in agreement with that of the standard solutions. These results confirmed that our Exo I-assisted signal amplification assay is a very promising innovative tool in the accurate analysis of antibiotics-based screening detection due to its outstanding analytical sensitivity.

\section{Conclusions}

In this study, we have successfully developed a label-free targetbinding induced aptamer structure switch colorimetric sensor for ultrasensitive detection of kanamycin based on Exo I-assisted signal amplification and polycationic protamine mediated aggregation of negatively charged AuNPs. This design took the full advantages of the outstanding signal amplification effect of Exo I, high binding affinity of aptamer with target as well as strong electrostatic interaction between protamine and negatively charged AuNPs, and thus successfully attained the ultrasensitive detection of kanamycin with detection limit of $2.8 \times$ $10^{-14} \mathrm{M}$. Our aptamer colorimetric assay only needs simple mixing of the dsDNA probe, target, protamine and AuNPs in homogeneous solution, without containing modification, or separation steps. Besides, the proposed strategy could be applied for detection of kanamycin in milk samples with high reliability. Due to these advantages, this method is promising for wide applications to analysis other small molecules and proteins.

\section{CRediT authorship contribution statement}

Jingwen Li: Conceptualization, Methodology, Investigation, Writing - original draft. Yongming Liu: Investigation, Writing - review \& editing. Hao Lin: Data curation, Validation. Yan Chen: Validation, Writing review \& editing. Zhenbo Liu: Data curation. Xuming Zhuang: Investigation. Chunyuan Tian: Validation. Xiuli Fu: Supervision, Funding acquisition, Writing - review \& editing. : . Lingxin Chen: Funding acquisition, Project administration, Supervision, Conceptualization, Writing - review \& editing.

\section{Declaration of Competing Interest}

The authors declare that they have no known competing financial interests or personal relationships that could have appeared to influence the work reported in this paper.

\section{Acknowledgements}

We gratefully appreciate the financial support from the National Natural Science Foundation of China (grant no. 21705139; 61801274; 21778047), the Natural Science Foundation of Shandong Province in China (grant no. ZR2017BB026; ZR2016CB25), the Qingchuang Science and Technology Program of Shandong Province in China (grant no. 2019KJF029), and Taishan Scholar Project Special Funding (No. ts20190962).

\section{Appendix A. Supplementary data}

Supplementary data to this article can be found online at https://doi. org/10.1016/j.foodchem.2020.128988.

\section{References}

Abnous, K., Danesh, N. M., Alibolandi, M., Ramezani, M., Sarreshtehdar Emrani, A., Zolfaghari, R., \& Taghdisi, S. M. (2017). A new amplified $\pi$-shape electrochemical aptasensor for ultrasensitive detection of aflatoxin $\mathrm{B}_{1}$. Biosensors and Bioelectronics, 94, 374-379. https://doi.org/10.1016/j.bios.2017.03.028.

Abu-Qare, A. W., \& Abou-Donia, M. B. (2001). Simultaneous determination of malathion, permethrin, DEET (N,N-diethyl-m-toluamide), and their metabolites in rat plasma and urine using high performance liquid chromatography. Journal of Pharmaceutical and Biomedical Analysis, 26(2), 291-299. https://doi.org/10.1016/S0731-7085(01) 00407-1.

Berijani, S., Assadi, Y., Anbia, M., Milani Hosseini, M.-R., \& Aghaee, E. (2006). Dispersive liquid-liquid microextraction combined with gas chromatography-flame photometric detection. Journal of Chromatography A, 1123(1), 1-9. https://doi.org/ 10.1016/j.chroma.2006.05.010.

Blanchaert, B., Poderós Jorge, E., Jankovics, P., Adams, E., \& Van Schepdael, A. (2013). Assay of kanamycin A by HPLC with direct UV detection. Chromatographia, 76(2122), 1505-1512. https://doi.org/10.1007/s10337-013-2440-8.

Cao, R., \& Li, B. X. (2011). A simple and sensitive method for visual detection of heparin using positively-charged gold nanoparticles as colorimetric probes. Chemical Communications, 47(10), 2865-2867. https://doi.org/10.1039/C0CC05094F.

Chen, X., Hong, F., Cao, Y., Hu, F., Wu, Y., Wu, D., Li, T., Lin, J., \& Gan, N. (2018). A microchip electrophoresis-based assay for ratiometric detection of kanamycin by R-shape probe and exonuclease-assisted signal amplification. Talanta, 189, 494-501. https://doi.org/10.1016/j.talanta.2018.07.010.

Chen, Z., Tan, Y., Zhang, C., Yin, L., Ma, H., Ye, N., .. Lin, Y. (2014). A colorimetric aptamer biosensor based on cationic polymer and gold nanoparticles for the ultrasensitive detection of thrombin. Biosensors and Bioelectronics, 56, 46-50. https://doi.org/10.1016/j.bios.2014.01.012.

Chen, Y. Q., Wang, Z. Q., Wang, Z. H., Tang, S. S., Zhu, Y., \& Xiao, X. L. (2008). Rapid enzyme-linked immunosorbent assay and colloidal gold immunoassay for kanamycin and tobramacin in swine tissues. Journal of Agricultural and Food Chemistry, 56(9), 2944-2952. https://doi.org/10.1021/jf703602b.

Chen, Z., Xiong, F., Yu, A., \& Lai, G. (2019). Aptamer biorecognition-triggered DNAzyme liberation and Exo III-assisted target recycling for ultrasensitive homogeneous colorimetric bioassay of kanamycin antibiotic. Chemical Communications, 55(27), 3959-3962. https://doi.org/10.1039/C8CC10107H.

García-Ruiz, C., Álvarez-Llamas, G., Puerta, Á., Blanco, E., Sanz-Medel, A., \& Marina, M. L. (2005). Enantiomeric separation of organophosphorus pesticides by capillary electrophoresis. Analytica Chimica Acta, 543(1-2), 77-83. https://doi.org/ 10.1016/j.aca.2005.04.027.

Han, S., Li, B., Song, Z., Pan, S., Zhang, Z., Yao, H., ... Xu, G. (2017). A kanamycin sensor based on an electrosynthesized molecularly imprinted poly-o-phenylenediamine film on a single-walled carbon nanohorn modified glassy carbon electrode. Analyst, 142 (1), 218-223. https://doi.org/10.1039/C6AN02338J.

Han, X., Yu, Z., Li, F., Shi, W., Fu, C., Yan, H., \& Zhang, G. (2019). Two kanamycin electrochemical aptamer-based sensors using different signal transduction mechanisms: A comparison of electrochemical behavior and sensing performance. Bioelectrochemistry, 129, 270-277. https://doi.org/10.1016/j. bioelechem.2019.06.004

Hao, L., Gu, H., Duan, N., Wu, S., \& Wang, Z. (2016). A chemiluminescent aptasensor for simultaneous detection of three antibiotics in milk. Analytical Methods, 8(44), 7929-7936. https://doi.org/10.1039/C6AY02304E. 
Hernández, M., Borrull, F., \& Calull, M. (2003). Analysis of antibiotics in biological samples by capillary electrophoresis. TrAC Trends in Analytical Chemistry, 22(7), 416-427. https://doi.org/10.1016/S0165-9936(03)00702-7.

Li, C., Zhang, Y., Eremin, S. A., Yakup, O., Yao, G., \& Zhang, X. (2017). Detection of kanamycin and gentamicin residues in animal-derived food using IgY antibody based ic-ELISA and FPIA. Food Chemistry, 227, 48-54. https://doi.org/10.1016/j. foodchem.2017.01.058.

Liu, R., Huang, Y., Ma, Y., Jia, S., Gao, M., Li, J., Zhang, H., Xu, D., Wu, M., Chen, Y., Zhu, Z., \& Yang, C. (2015). Design and synthesis of target-responsive aptamer-crosslinked hydrogel for visual quantitative detection of ochratoxin A. ACS Applied Materials \& Interfaces, 7(12), 6982-6990. https://doi.org/10.1021/acsami.5b01120.

Liu, H., Li, L., Duan, L., Wang, X., Xie, Y., Tong, L., ... Tang, B. (2013). High specific and ultrasensitive isothermal detection of MicroRNA by padlock probe-based exponential rolling circle amplification. Analytical Chemistry, 85(16), 7941-7947. https://doi.org/10.1021/ac401715k.

Liu, J., \& Lu, Y. (2006). Preparation of aptamer-linked gold nanoparticle purple aggregates for colorimetric sensing of analytes. Nature Protocols, 1(1), 246-252. https://doi.org/10.1038/nprot.2006.38.

Long, F., Zhang, Z., Yang, Z., Zeng, J., \& Jiang, Y. (2015). Imprinted electrochemical sensor based on magnetic multi-walled carbon nanotube for sensitive determination of kanamycin. Journal of Electroanalytical Chemistry, 755, 7-14. https://doi.org/ 10.1016/j.jelechem.2015.07.018.

Luo, Y., Xu, J., Li, Y., Gao, H., Guo, J., Shen, F., \& Sun, C. (2015). A novel colorimetric aptasensor using cysteamine-stabilized gold nanoparticles as probe for rapid and specific detection of tetracycline in raw milk. Food Control, 54, 7-15. https://doi org/10.1016/j.foodcont.2015.01.005.

Ma, L., Sun, N., Tu, C., Zhang, Q., \& Diao, A. (2017). Design of an aptamer - based fluorescence displacement biosensor for selective and sensitive detection of kanamycin in aqueous samples. RSC Advances, 7(61), 38512-38518. https://doi. org/10.1039/C7RA07052G.

Ma, R.-N., Wang, L.-L., Wang, H.-F., Jia, L.-P., Zhang, W., Shang, L., Xue, Q.-W., Jia, W.L., Liu, Q.-Y., \& Wang, H.-S. (2018). Highly sensitive ratiometric electrochemical DNA biosensor based on homogeneous exonuclease III-assisted target recycling amplification and one-step triggered dual-signal output. Sensors and Actuators B: Chemical, 269, 173-179. https://doi.org/10.1016/j.snb.2018.04.143.

Obare, S. O., Hollowell, R. E., \& Murphy, C. J. (2002). Sensing strategy for lithium ion based on gold nanoparticles. Langmuir, 18(26), 10407-10410. https://doi.org/ 10.1021/la0260335.

Qin, L., Zeng, G., Lai, C., Huang, D., Zhang, C., Xu, P., Hu, T., Liu, X., Cheng, M., Liu, Y., Hu, L., \& Zhou, Y. (2017). A visual application of gold nanoparticles: Simple, reliable and sensitive detection of kanamycin based on hydrogen-bonding recognition. Sensors and Actuators B: Chemical, 243, 946-954. https://doi.org/10.1016/j. snb.2016.12.086.

Ramezani, M., Danesh, N. M., Lavaee, P., Abnous, K., \& Taghdisi, S. M. (2016). A selective and sensitive fluorescent aptasensor for detection of kanamycin based on catalytic recycling activity of exonuclease III and gold nanoparticles. Sensors and Actuators B: Chemical, 222, 1-7. https://doi.org/10.1016/j.snb.2015.08.024.

Sabela, M., Balme, S., Bechelany, M., Janot, J.-M., \& Bisetty, K. (2017). A review of gold and silver nanoparticle-based colorimetric sensing assays. Advanced Engineering Materials, 19(12), 1700270. https://doi.org/10.1002/adem.201700270.

Saratale, G. D., Saratale, R. G., Ghodake, G., Shinde, S., Kim, D. Y., Alyousef, A. A., .. Shin, H. S. (2020). Chlortetracycline-functionalized silver nanoparticles as a colorimetric probe for aminoglycosides: ultrasensitive determination of kanamycin and streptomycin. Nanomaterials, 10(5), 997. https://doi.org/10.3390/ nano10050997.

Shahdordizadeh, M., Yazdian-Robati, R., Ansari, N., Ramezani, M., Abnous, K., \& Taghdisi, S. M. (2018). An aptamer-based colorimetric lead (II) assay based on the use of gold nanoparticles modified with dsDNA and exonuclease I. Microchimica Acta, 185(2), 151. https://doi.org/10.1007/s00604-018-2699-4.

Tu, M. H., Sun, T., \& Grattan, K. T. V. (2014). LSPR optical fibre sensors based on hollow gold nanostructures. Sensors and Actuators B: Chemical, 191, 37-44. https://doi.org/ 10.1016/j.snb.2013.09.094.

Wang, C., Chen, D., Wang, Q., \& Tan, R. (2017). Kanamycin detection based on the catalytic ability enhancement of gold nanoparticles. Biosensors and Bioelectronics, 91, 262-267. https://doi.org/10.1016/j.bios.2016.12.042.

Wang, C., Wang, C., Wang, Q., \& Chen, D. (2017). Resonance light scattering method for detecting kanamycin in milk with enhanced sensitivity. Analytical and Bioanalytical Chemistry, 409(11), 2839-2846. https://doi.org/10.1007/s00216-017-0228-7.

Xing, X.-J., Xiao, W.-L., Liu, X.-G., Zhou, Y., Pang, D.-W., \& Tang, H.-W. (2016). A fluorescent aptasensor using double-stranded DNA/graphene oxide as the indicator probe. Biosensors and Bioelectronics, 78, 431-437. https://doi.org/10.1016/ j.bios.2015.11.076.

Xu, Y., Han, T., Li, X., Sun, L., Zhang, Y., \& Zhang, Y. (2015). Colorimetric detection of kanamycin based on analyte-protected silver nanoparticles and aptamer-selective sensing mechanism. Analytica Chimica Acta, 891, 298-303. https://doi.org/10.1016/ j.aca.2015.08.013.

Xu, C. N., Ying, Y. B., \& Ping, J. F. (2019). Colorimetric aggregation assay for kanamycin using gold nanoparticles modified with hairpin DNA probes and hybridization chain reaction-assisted amplification. Microchimica Acta, 186(7), 448. https://doi.org/ 10.1007/s00604-019-3574-7.

Yang, C. J., Jockusch, S., Vicens, M., Turro, N. J., \& Tan, W. (2005). Light-switching excimer probes for rapid protein monitoring in complex biological fluids. Proceedings of the National Academy of Sciences, 102(48), 17278-17283. https://doi.org/ 10.1073/pnas.0508821102.

Yu, S., Wei, Q., Du, B., Wu, D., Li, H., Yan, L., ... Zhang, Y. (2013). Label-free immunosensor for the detection of kanamycin using $\mathrm{Ag} @ \mathrm{Fe}_{3} \mathrm{O}_{4}$ nanoparticles and thionine mixed graphene sheet. Biosensors and Bioelectronics, 48, 224-229. https:// doi.org/10.1016/j.bios.2013.04.025.

Zeng, R., Tang, Y., Zhang, L., Luo, Z., \& Tang, D. (2018). Dual-readout aptasensing of antibiotic residues based on gold nanocluster-functionalized $\mathrm{MnO}_{2}$ nanosheets with target-induced etching reaction. Journal of Materials Chemistry B, 6(48), 8071-8077. https://doi.org/10.1039/C8TB02642D.

Zhang, H., Fan, M., Jiang, J., Shen, Q., Cai, C., \& Shen, J. (2019). Sensitive electrochemical biosensor for MicroRNAs based on duplex-specific nuclease-assisted target recycling followed with gold nanoparticles and enzymatic signal amplification. Analytica Chimica Acta, 1064, 33-39. https://doi.org/10.1016/j. aca.2019.02.060.

Zhou, N. D., Zhang, J., \& Tian, Y. P. (2014). Aptamer-based spectrophotometric detection of kanamycin in milk. Analytical Methods, 6(5), 1569-1574. https://doi.org/ 10.1039/C3AY41816B.

Zhu, Y.e., Chandra, P., Song, K.-M., Ban, C., \& Shim, Y.-B. (2012). Label-free detection of kanamycin based on the aptamer-functionalized conducting polymer/gold nanocomposite. Biosensors and Bioelectronics, 36(1), 29-34. https://doi.org/ 10.1016/j.bios.2012.03.034. 